Acta Crystallographica Section E

Structure Reports

Online

ISSN 1600-5368

\section{3-Azido-3-deoxy-2,2':5,6-di-O-isopropylidene- 2-C-hydroxmethyl-D-gulono-1,4-lactone}

The title azidolactone, $\mathrm{C}_{13} \mathrm{H}_{19} \mathrm{O}_{6} \mathrm{~N}_{3}$, formed by $\mathrm{S}_{\mathrm{N}} 2$ displacement of the trifluoromethanesulfonate with sodium azide, is the first example of a branched $\beta$-sugar amino acid scaffold.

\section{Comment}

Department of Chemical Crystallography, Chemical Research Laboratory, Oxford University, Mansfield Road, Oxford OX1 3TA, England, and ${ }^{\mathbf{b}}$ Department of Organic Chemistry, Chemical Research Laboratory, Oxford University, Mansfield Road, Oxford OX1 3TA, England

Correspondence e-mail: christopher.harding@seh.ox.ac.uk

\footnotetext{
Key indicators

Single-crystal X-ray study

$T=120 \mathrm{~K}$

Mean $\sigma(\mathrm{C}-\mathrm{C})=0.005 \AA$

$R$ factor $=0.045$

$w R$ factor $=0.108$

Data-to-parameter ratio $=9.3$
}

For details of how these key indicators were automatically derived from the article, see http://journals.iucr.org/e.

Gellman (Lai \& Gellman, 2003; Hayen et al., 2004) and Seebach (Lelais \& Seebach, 2003; Rueping et al., 2004) have pioneered studies on the exploitation of $\beta$-amino acids in their adoption of secondary structural features in short chains. Although sugar amino acids (SAA) have been extensively investigated as peptidomimetics and dipeptide isosteres (Schweizer, 2002; Chakraborty et al., 2004), there have been very few studies of $\beta$-SAAs (Jenkinson \& Fleet, 2004; Johnson et al., 2004), even though some oxetane-derived $\beta$-SAAs exhibited novel helical structures (Barker et al., 2001). This paper reports the structure of the $\beta$-azidolactone, (4), which is a novel $\beta$-SAA scaffold containing a branched carbon chain.
(C) 2004 International Union of Crystallography Printed in Great Britain - all rights reserved

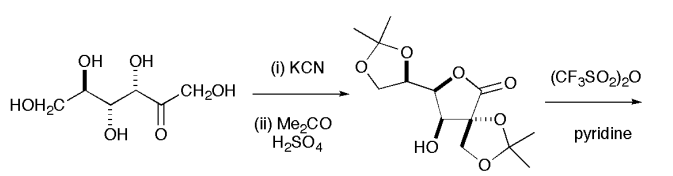

(1)

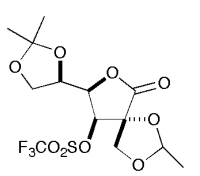

(3)
Received 22 October 2004 Accepted 10 November 2004 Online 20 November 2004
D-Fructose may be readily converted into the diacetonide (2) (Hotchkiss et al., 2004); esterification of (2) with triflic anhydride gave the corresponding stable trifluoromethanesulfonate. Reaction of (3) with sodium azide in DMF gave an organic azide in good yield. The structure of this azide is fraught with uncertainties; the trifluoromethanesulfonate in (3) has two $\beta$-O atoms and the adjacent $\alpha-\mathrm{C}$ atom is trisubstituted, so the efficiency of the $\mathrm{S}_{\mathrm{N}} 2$ reaction is surprising. There is considerable ambiguity in the stereochemistry of the product, since there may well be neighbouring group participation by the $\mathrm{O}$ atom; it is also possible that some rearrangement that maintained the same connectivity of $\mathrm{CH}$ atoms may have occurred. However, X-ray crystallographic analysis of the product of the reaction showed that the anticipated inverted azide, (4), had indeed been produced.

\section{Experimental}

The full preparative method is not available for publication as yet. The sample was crystallized from diethyl ether by inward diffusion of $n$-hexane to give lath-shaped colourless crystals. 


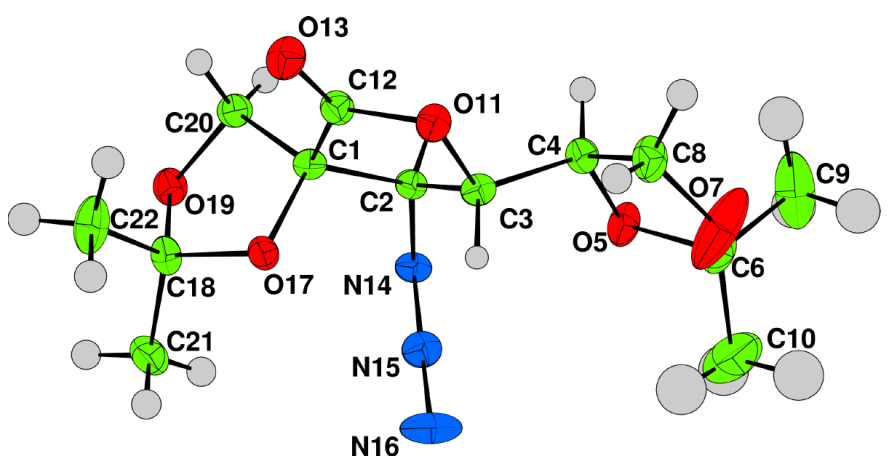

Figure 1

The title molecule at $120 \mathrm{~K}$, with displacement ellipsoids drawn at the $50 \%$ probability level. The large ellipsoids at atoms $07, \mathrm{C} 9$ and $\mathrm{C} 10$ are discussed in the text. $\mathrm{H}$ atoms are shown as spheres of arbitrary radii.

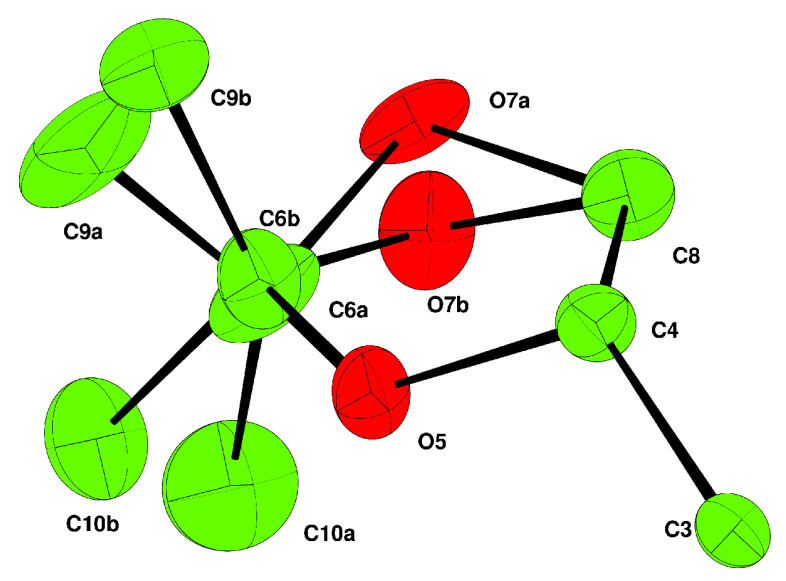

Figure 2

The disordered fragment of the title molecule at $120 \mathrm{~K}$, displayed as a 'split atom' model. Even with firm anisotropic displacement parameter similarity restraints, the ellipsoids do not conform to any reasonable physical model.

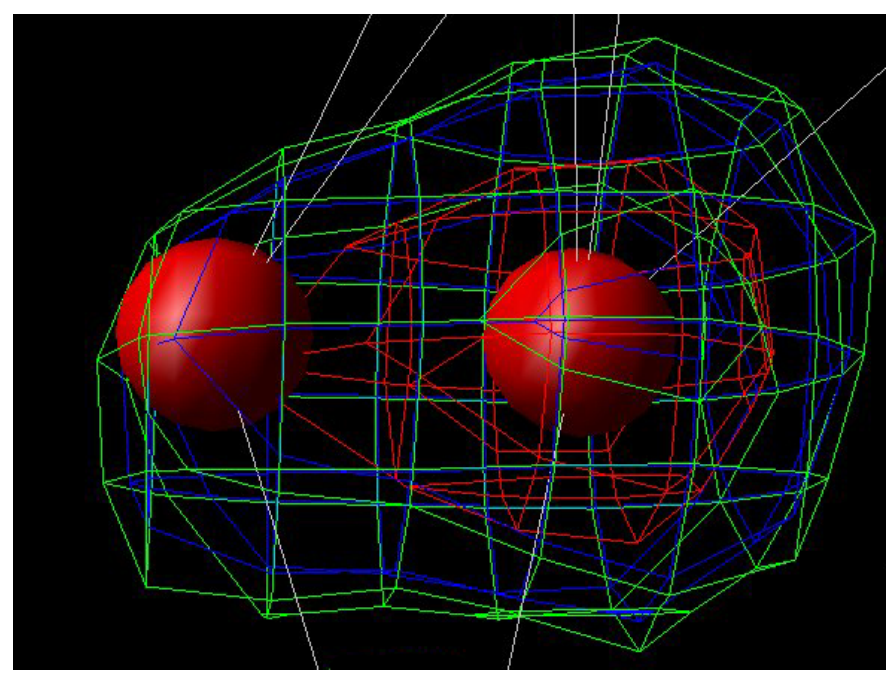

Figure 3

$F_{\mathrm{o}}$ electron density map viewed perpendicular to the line through atoms $\mathrm{O} 7 a$ and $\mathrm{O} 7 b$, computed excluding phasing information derived from these two partial atoms. There are no distinct lobes near the two atom positions proposed by the 'split atom' refinement, suggesting that there still remains substantial dynamic disorder.

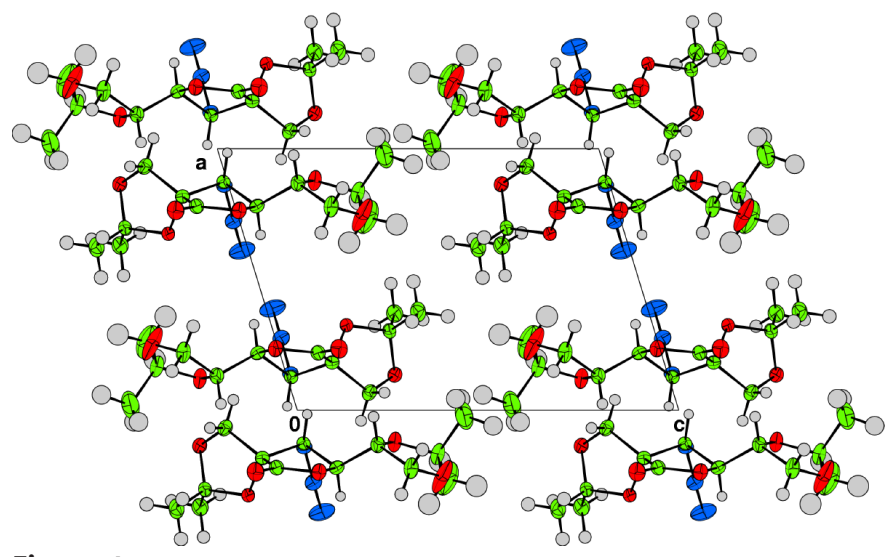

Figure 4

Packing diagram of the title compound, viewed along the $b$ axis.

$\begin{array}{ll}\text { Crystal data } & \\ \mathrm{C}_{13} \mathrm{H}_{19} \mathrm{~N}_{3} \mathrm{O}_{6} & D_{x}=1.326 \mathrm{Mg} \mathrm{m}^{-3} \\ M_{r}=313.31 & \text { Mo } K \alpha \text { radiation } \\ \text { Monoclinic, } P 2_{1} & \text { Cell parameters from } 1734 \\ a=8.7755(2) \AA & \quad \text { reflections } \\ b=7.6452(2) \AA & \theta=5-27^{\circ} \\ c=12.2232(3) \AA & \mu=0.11 \mathrm{~mm}^{-1} \\ \beta=106.9659(11)^{\circ} & T=120 \mathrm{~K} \\ V=784.37(3) \AA^{3} & \text { Lath, colourless } \\ Z=2 & 0.30 \times 0.20 \times 0.10 \mathrm{~mm}\end{array}$

Data collection

Nonius KappaCCD diffractometer $\omega$ scans

Absorption correction: multi-scan (DENZO/SCALEPACK;

Otwinowski \& Minor, 1997)

$T_{\min }=0.98, T_{\max }=0.99$

3320 measured reflections

Refinement

Refinement on $F^{2}$

$R\left[F^{2}>2 \sigma\left(F^{2}\right)\right]=0.045$

$w R\left(F^{2}\right)=0.109$

$S=0.93$

1866 reflections

200 parameters

H-atom parameters constrained

1869 independent reflections 1655 reflections with $I>2 \sigma(I)$

$R_{\text {int }}=0.015$

$\theta_{\max }=27.5^{\circ}$

$h=-11 \rightarrow 11$

$k=-9 \rightarrow 9$

$l=-15 \rightarrow 15$
$w=1 /\left[\sigma^{2}(F)+(0.0522 p)^{2}+0.571 p\right]$,
where $p=\left[\max \left(F_{o}^{2}, 0\right)+2 F_{c}^{2}\right] / 3$
$(\Delta / \sigma)_{\max }<0.001$
$\Delta \rho_{\max }=0.65 \mathrm{e}^{-3}$
$\Delta \rho_{\min }=-0.42 \mathrm{e}^{-3}$
Extinction correction: Larson (1970)
Extinction coefficient: $3.0(5) \times 10^{2}$

Table 1

Selected interatomic distances $(\AA)$.

\begin{tabular}{llll}
\hline $\mathrm{O} 5-\mathrm{C} 6$ & $1.433(3)$ & $\mathrm{C} 6-\mathrm{C} 10$ & $1.486(7)$ \\
$\mathrm{C} 6-\mathrm{O} 7$ & $1.370(5)$ & $\mathrm{O} 7-\mathrm{C} 8$ & $1.384(4)$ \\
$\mathrm{C} 6-\mathrm{C} 9$ & $1.503(6)$ & & \\
\hline
\end{tabular}

An initial data set was collected at $190 \mathrm{~K}$. This gave reasonable refinement $\left[N_{\text {measured }}=2766, R_{\text {int }}=0.02, N_{\text {ref }}=1748, R_{w}(2 \sigma)=0.081\right.$, $R(2 \sigma)=0.034]$, though atoms $\mathrm{O} 7, \mathrm{C} 9$ and $\mathrm{C} 10$ had very elongated displacement ellipsoids. It was unclear whether the $\mathrm{C} 6 / \mathrm{O} 7 / \mathrm{C} 9 / \mathrm{C} 10$ fragment should be modelled with large anisotropic displacement parameters (ADPs) or with 'split atoms'. Refinement was continued with this fragment represented by 'split atoms'. However, the ADPs could not be explained by a rational physical model. As with the unsplit model, the bond lengths deviated unacceptably from averages drawn by MOGUL (Bruno et al., 2004) from the Cambridge Structural Database (CSD; Version 5.25; Allen, 2002), even with the application of firm bond length similarity restraints. 
In order to resolve this issue, data were recollected at $120 \mathrm{~K}$. It is the result of this refinement that is reported in the CIF. Even at this temperature, the ADPs of the problematic group remained large, though not as large as in the $190 \mathrm{~K}$ data set. Both the large ADP and the 'split atom' refinements continued to give unacceptable bond lengths and ellipsoids (see Fig. 2). Fig. 3, plotted with $M C E$ (Hušák \& Kratochvíl, 2003), shows the observed electron density perpendicular to the line connecting atoms $\mathrm{O} 7 a$ and $\mathrm{O} 7 b$. The map is phased by all of the structure except these two atoms, which are included for illustrative purposes at the positions they refine to in the 'split atom' model. There is a smooth transition in electron density between the two sites, with no evident build-up of density at either site.

One interpretation of these observations is that neither the split atom model nor the large ADP model really represents what is occurring in this structure. It is clearly something more complicated than simply having the envelope flap (O7/O7a) distributed over two sites on opposite sides of a plane through the other ring atoms. Of 29 structures in the CSD containing this moiety some clearly have the atom corresponding to $\mathrm{O} 7$ as the 'flap', some have $\mathrm{C} 6$ as the flap, but there are also a number in which no four atoms form a convincing plane. It seems that, even in the solid state, there is a continuum between an O-flap and a C-flap geometry. The amount of material available was insufficient to enable low-temperature solid-state NMR measurements to be carried out.

For the large ADP model, all $\mathrm{H}$ atoms were seen in the difference electron density map (even those on the highly anisotropic C9 and $\mathrm{C} 10)$. Their positions and $U_{\text {iso }}(\mathrm{H})$ values were regularized by several cycles of refinement using slack restraints, after which the refinement was completed using 'riding' constraints and all reflections with $I>-3 \sigma(I)$. Fig. 4 is a packing diagram showing an alternation of highly mobile and highly ordered groups lying in a plane at approximately $c / 2$.

Data collection: COLLECT (Nonius, 1997-2001); cell refinement: DENZO/SCALEPACK (Otwinowski \& Minor, 1997); data reduction: DENZO/SCALEPACK; program(s) used to solve structure: SIR92 (Altomare et al., 1994); program(s) used to refine structure: CRYSTALS (Betteridge et al., 2003); molecular graphics:
CAMERON (Watkin et al., 1996); software used to prepare material for publication: CRYSTALS.

Financial support (to RS) provided through the European Community's Human Potential Programme under contract HPRN-CT-2002-00173 is gratefully acknowledged.

\section{References}

Allen, F. H. (2002). Acta Cryst. B58, 380-388.

Altomare, A., Cascarano, G., Giacovazzo, C., Guagliardi, A., Burla, M. C., Polidori, G. \& Camalli, M. (1994). J. Appl. Cryst. 27, 435.

Barker, S. F., Angus, D., Taillefumier, C., Probert, M. R., Watkin, D. J., Watterson, M. P., Claridge, T. D. W., Hungerford, N. L. \& Fleet, G. W. J. (2001). Tetrahedron Lett. 42, 4247-4250.

Betteridge, P. W., Carruthers, J. R., Cooper, R. I., Prout, K. \& Watkin, D. J. (2003). J. Appl. Cryst. 36, 1487.

Bruno, I. J., Cole, J. C., Kessler, M., Luo, J., Motherwell, W. D. S., Purkis, L. H., Smith, B. R., Taylor, R., Cooper, R. I., Harris, S. E. \& Orpen, A. G. (2004). J. Chem. Inf. Comput. Sci. In the press.

Chakraborty, T. K., Srinivasi, P., Tapadar, S. \& Mohan, B. K. (2004). J. Chem. Sci. 116, 187-207.

Hayen, A., Schmitt, M. A., Ngassa, F. N., Thomasson, K. A. \& Gellman, S. H. (2004). Angew. Chem. Int. Ed. 43, 505-510.

Hotchkiss, D., Soengas, R., Simone, M. I., van Ameijde, J., Hunter, S., Cowley, A. R. \& Fleet, G. W. J. (2004). Tetrahedron Lett. 45. In the press.

Hušák, M. \& Kratochvíl, B. (2003). J. Appl. Cryst. 36, 1104.

Jenkinson, S. F. \& Fleet, G. W. J. (2004). Tetrahedron Asymmetry, 15, $2667-$ 2679.

Johnson, S. W., Jenkinson, S. F., Angus, D., Taillefumier, C., Jones, J. H. \& Fleet, G. W. J. (2004). Tetrahedron Asymmetry, 15, 2681-2686.

Lai, J. R. \& Gellman, S. H. (2003). Protein Sci. 12, 560-566.

Larson, A. C. (1970). Crystallographic Computing, edited by F. R. Ahmed, S. R. Hall \& C. P. Huber, pp. 291-294. Copenhagen: Munksgaard.

Lelais, G. \& Seebach, D. (2003). Helv. Chim. Acta, 86, 4152-4168.

Nonius (1997-2001). COLLECT. Nonius BV, Delft, The Netherlands.

Otwinowski, Z. \& Minor, W. (1997). Methods in Enzymology, Vol. 276, Macromolecular Crystallography, Part A, edited by C. W. Carter Jr \& R. M. Sweet, pp. 307-326. New York: Academic Press.

Rueping, M., Mahajan, Y. R., Jaun, B. \& Seebach, D. (2004). Chem. Eur. J. 10, 1607-1615.

Schweizer, F. (2002). Angew. Chem. Int. Ed. 41, 230-253.

Watkin, D. J., Prout, C. K. \& Pearce, L. J. (1996). CAMERON. Chemical Crystallography Laboratory, Oxford, England. 


\section{supporting information}

Acta Cryst. (2004). E60, o2334-o2336 [https://doi.org/10.1107/S1600536804029113]

\section{3-Azido-3-deoxy-2,2':5,6-di-O-isopropylidene-2-C-hydroxmethyl-D-gulono-1,4- lactone}

\section{Christopher C. Harding, Howard Shallard-Brown, David J. Watkin, Raquel Soengas and George}

W. J. Fleet

3-Azido-3-deoxy-2,2':5,6-di-O-isopropylidene-2-C-hydroxmethyl-D- gulono-1,4-lactone

Crystal data

$\mathrm{C}_{13} \mathrm{H}_{19} \mathrm{~N}_{3} \mathrm{O}_{6}$

$M_{r}=313.31$

Monoclinic, $P 2_{1}$

$a=8.7755(2) \AA$

$b=7.6452(2) \AA$

$c=12.2232(3) \AA$

$\beta=106.9659(11)^{\circ}$

$V=784.37(3) \AA^{3}$

$Z=2$

\section{Data collection}

Nonius KappaCCD

diffractometer

Graphite monochromator

$\omega$ scans

Absorption correction: multi-scan

DENZO/SCALEPACK (Otwinowski \& Minor, 1997)

$T_{\min }=0.98, T_{\max }=0.99$

Refinement

Refinement on $F^{2}$

Least-squares matrix: full

$R\left[F^{2}>2 \sigma\left(F^{2}\right)\right]=0.045$

$w R\left(F^{2}\right)=0.109$

$S=0.93$

1866 reflections

200 parameters

1 restraint

Primary atom site location: structure-invariant direct methods
$F(000)=332$

$D_{\mathrm{x}}=1.326 \mathrm{Mg} \mathrm{m}^{-3}$

Mo $K \alpha$ radiation, $\lambda=0.71073 \AA$

Cell parameters from 1734 reflections

$\theta=5-27^{\circ}$

$\mu=0.11 \mathrm{~mm}^{-1}$

$T=120 \mathrm{~K}$

Plate, colourless

$0.30 \times 0.20 \times 0.10 \mathrm{~mm}$

3320 measured reflections

1869 independent reflections

1655 reflections with $I>2 \sigma(I)$

$R_{\text {int }}=0.015$

$\theta_{\text {max }}=27.5^{\circ}, \theta_{\text {min }}=5.2^{\circ}$

$h=-11 \rightarrow 11$

$k=-9 \rightarrow 9$

$l=-15 \rightarrow 15$

Hydrogen site location: inferred from neighbouring sites

$\mathrm{H}$-atom parameters constrained

Method: SHELXL97 (Sheldrick, 1997); $w=$

$1 /\left[\sigma^{2}(\mathrm{f})+(0.0522 \mathrm{p})^{2}+0.571 \mathrm{p}\right]$,

where $\mathrm{p}=\left[\max \left(F_{0}^{2}, 0\right)+2 F_{\mathrm{c}}{ }^{2}\right] / 3$

$(\Delta / \sigma)_{\max }=0.000326$

$\Delta \rho_{\max }=0.65$ e $\AA^{-3}$

$\Delta \rho_{\min }=-0.42$ e $\AA^{-3}$

Extinction correction: Larson 1970

Crystallographic Computing eq 22

Extinction coefficient: 300 (50) 
Fractional atomic coordinates and isotropic or equivalent isotropic displacement parameters $\left(\AA^{2}\right)$

\begin{tabular}{|c|c|c|c|c|}
\hline & $x$ & $y$ & $z$ & $U_{\text {iso }} * / U_{\text {eq }}$ \\
\hline $\mathrm{C} 1$ & 0.8173 & $0.1205(4)$ & $0.8704(2)$ & 0.0207 \\
\hline $\mathrm{C} 2$ & 0.8728 & $0.0356(4)$ & $0.9895(2)$ & 0.0197 \\
\hline $\mathrm{C} 3$ & 0.7808 & $0.1387(4)$ & $1.0574(2)$ & 0.0214 \\
\hline $\mathrm{C} 4$ & $0.8684(3)$ & $0.1553(4)$ & $1.1835(2)$ & 0.0253 \\
\hline $\mathrm{O} 5$ & 0.8788 & $-0.0165(3)$ & $1.22982(16)$ & 0.0306 \\
\hline C6 & $0.8328(4)$ & $-0.0098(5)$ & $1.3330(3)$ & 0.0349 \\
\hline $\mathrm{O} 7$ & $0.7463(5)$ & $0.1409(5)$ & $1.3271(3)$ & 0.0777 \\
\hline $\mathrm{C} 8$ & $0.7788(4)$ & $0.2612(4)$ & $1.2524(3)$ & 0.0345 \\
\hline $\mathrm{C} 9$ & $0.9801(6)$ & $0.0021(9)$ & $1.4333(3)$ & 0.0762 \\
\hline $\mathrm{C} 10$ & $0.7390(8)$ & $-0.1702(9)$ & $1.3373(5)$ & 0.0891 \\
\hline O11 & $0.7642(2)$ & $0.3122(3)$ & $1.00698(16)$ & 0.0251 \\
\hline $\mathrm{C} 12$ & $0.7805(3)$ & $0.3076(4)$ & $0.8996(2)$ & 0.0234 \\
\hline $\mathrm{O} 13$ & 0.7649 & 0.4350 & $0.84141(18)$ & 0.0315 \\
\hline N14 & $0.8584(3)$ & $-0.1555(3)$ & $0.9887(2)$ & 0.0219 \\
\hline N15 & 0.7245 & $-0.2124(4)$ & $0.9845(2)$ & 0.0267 \\
\hline N16 & $0.6106(4)$ & $-0.2828(4)$ & $0.9831(3)$ & 0.0454 \\
\hline $\mathrm{O} 17$ & $0.6748(2)$ & $0.0412(3)$ & $0.80306(15)$ & 0.0223 \\
\hline C18 & $0.6968(3)$ & $-0.0131(4)$ & $0.6948(2)$ & 0.0246 \\
\hline O19 & $0.8643(2)$ & $-0.0228(3)$ & $0.71573(16)$ & 0.0250 \\
\hline $\mathrm{C} 20$ & $0.9322(3)$ & $0.1103(4)$ & $0.7966(2)$ & 0.0252 \\
\hline $\mathrm{C} 21$ & $0.6301(4)$ & $-0.1943(5)$ & $0.6674(3)$ & 0.0338 \\
\hline $\mathrm{C} 22$ & $0.6235(4)$ & $0.1233(5)$ & $0.6046(3)$ & 0.0408 \\
\hline $\mathrm{H} 21$ & 0.9848 & 0.0640 & 1.0229 & $0.0240^{*}$ \\
\hline H31 & 0.6712 & 0.0884 & 1.0445 & $0.0251^{*}$ \\
\hline H41 & 0.9753 & 0.2039 & 1.1948 & $0.0304^{*}$ \\
\hline H81 & 0.8497 & 0.3528 & 1.2967 & $0.0438 *$ \\
\hline H82 & 0.6789 & 0.3123 & 1.2055 & $0.0432 *$ \\
\hline H91 & 0.9408 & 0.0093 & 1.5009 & $0.0912 *$ \\
\hline H92 & 1.0438 & -0.1057 & 1.4370 & $0.0913^{*}$ \\
\hline H93 & 1.0444 & 0.1073 & 1.4251 & $0.0920^{*}$ \\
\hline H101 & 0.7105 & -0.1659 & 1.4094 & $0.1170^{*}$ \\
\hline H102 & 0.8053 & -0.2750 & 1.3328 & $0.1173^{*}$ \\
\hline H103 & 0.6413 & -0.1563 & 1.2703 & $0.1167 *$ \\
\hline H201 & 1.0400 & 0.0778 & 0.8411 & $0.0321 *$ \\
\hline H202 & 0.9326 & 0.2218 & 0.7573 & $0.0317 *$ \\
\hline H211 & 0.6543 & -0.2349 & 0.5979 & $0.0399 *$ \\
\hline H212 & 0.6785 & -0.2742 & 0.7300 & $0.0403 *$ \\
\hline $\mathrm{H} 213$ & 0.5151 & -0.1924 & 0.6528 & $0.0409^{*}$ \\
\hline H221 & 0.6405 & 0.1021 & 0.5300 & $0.0487^{*}$ \\
\hline H 222 & 0.6603 & 0.2431 & 0.6279 & $0.0489^{*}$ \\
\hline $\mathrm{H} 223$ & 0.5076 & 0.1206 & 0.5922 & $0.0491 *$ \\
\hline
\end{tabular}


Atomic displacement parameters $\left(\AA^{2}\right)$

\begin{tabular}{lllllll}
\hline & $U^{11}$ & $U^{22}$ & $U^{33}$ & $U^{12}$ & $U^{13}$ & $U^{23}$ \\
\hline C1 & $0.0206(13)$ & $0.0191(13)$ & $0.0222(12)$ & $-0.0012(11)$ & $0.0061(10)$ & $-0.0002(11)$ \\
C2 & $0.0206(13)$ & $0.0182(13)$ & $0.0199(12)$ & $-0.0019(11)$ & $0.0051(10)$ & $-0.0009(10)$ \\
C3 & $0.0215(13)$ & $0.0186(15)$ & $0.0256(13)$ & $0.0009(11)$ & $0.0090(10)$ & $-0.0002(11)$ \\
C4 & $0.0289(15)$ & $0.0252(16)$ & $0.0223(12)$ & $-0.0017(12)$ & $0.0084(11)$ & $-0.0032(12)$ \\
O5 & $0.0445(12)$ & $0.0282(11)$ & $0.0235(9)$ & $0.0034(10)$ & $0.0166(9)$ & $0.0017(9)$ \\
C6 & $0.0495(19)$ & $0.0342(17)$ & $0.0279(14)$ & $-0.0014(17)$ & $0.0220(13)$ & $0.0011(14)$ \\
O7 & $0.118(3)$ & $0.069(2)$ & $0.080(2)$ & $0.051(2)$ & $0.082(2)$ & $0.0350(19)$ \\
C8 & $0.0481(19)$ & $0.0326(18)$ & $0.0262(14)$ & $0.0028(15)$ & $0.0161(14)$ & $-0.0044(13)$ \\
C9 & $0.086(3)$ & $0.109(5)$ & $0.0317(18)$ & $0.019(4)$ & $0.013(2)$ & $-0.001(3)$ \\
C10 & $0.120(5)$ & $0.089(5)$ & $0.080(4)$ & $-0.047(4)$ & $0.064(4)$ & $-0.009(3)$ \\
O11 & $0.0307(11)$ & $0.0208(11)$ & $0.0253(9)$ & $0.0038(9)$ & $0.0106(8)$ & $-0.0001(8)$ \\
C12 & $0.0244(14)$ & $0.0229(15)$ & $0.0225(12)$ & $-0.0005(12)$ & $0.0063(10)$ & $-0.0013(12)$ \\
O13 & $0.0395(12)$ & $0.0238(11)$ & $0.0315(11)$ & $0.0066(10)$ & $0.0106(9)$ & $0.0059(9)$ \\
N14 & $0.0187(12)$ & $0.0206(12)$ & $0.0266(11)$ & $-0.0017(10)$ & $0.0069(9)$ & $-0.0019(10)$ \\
N15 & $0.0279(13)$ & $0.0217(12)$ & $0.0320(13)$ & $0.0018(11)$ & $0.0109(10)$ & $-0.0010(10)$ \\
N16 & $0.0334(15)$ & $0.0299(16)$ & $0.078(2)$ & $-0.0116(13)$ & $0.0236(15)$ & $-0.0071(16)$ \\
O17 & $0.0209(9)$ & $0.0272(11)$ & $0.0189(8)$ & $-0.0018(8)$ & $0.0057(7)$ & $-0.0032(8)$ \\
C18 & $0.0245(13)$ & $0.0301(15)$ & $0.0186(11)$ & $0.0025(13)$ & $0.0053(10)$ & $-0.0021(12)$ \\
O19 & $0.0254(10)$ & $0.0270(11)$ & $0.0236(9)$ & $0.0018(9)$ & $0.0089(7)$ & $-0.0026(8)$ \\
C20 & $0.0287(14)$ & $0.0239(14)$ & $0.0259(13)$ & $-0.0041(13)$ & $0.0125(11)$ & $-0.0043(12)$ \\
C21 & $0.0341(16)$ & $0.0377(19)$ & $0.0289(14)$ & $-0.0073(15)$ & $0.0084(12)$ & $-0.0114(14)$ \\
C22 & $0.0436(18)$ & $0.054(2)$ & $0.0262(14)$ & $0.0194(18)$ & $0.0116(13)$ & $0.0104(15)$ \\
& & & & & & \\
& & & & & & \\
& & & & & & \\
& & & & &
\end{tabular}

Geometric parameters $\left(\AA,{ }^{\circ}\right)$

\begin{tabular}{llll}
\hline $\mathrm{C} 1-\mathrm{C} 2$ & $1.538(4)$ & $\mathrm{C} 9-\mathrm{H} 93$ & 1.004 \\
$\mathrm{C} 1-\mathrm{C} 12$ & $1.531(4)$ & $\mathrm{C} 10-\mathrm{H} 101$ & 0.984 \\
$\mathrm{C} 1-\mathrm{O} 17$ & $1.417(3)$ & $\mathrm{C} 10-\mathrm{H} 102$ & 1.001 \\
$\mathrm{C} 1-\mathrm{C} 20$ & $1.539(3)$ & $\mathrm{C} 10-\mathrm{H} 103$ & $1.362(3)$ \\
$\mathrm{C} 2-\mathrm{C} 3$ & $1.533(4)$ & $\mathrm{O} 11-\mathrm{C} 12$ & $1.190(4)$ \\
$\mathrm{C} 2-\mathrm{N} 14$ & $1.466(4)$ & $\mathrm{C} 12-\mathrm{O} 13$ & $1.240(3)$ \\
$\mathrm{C} 2-\mathrm{H} 21$ & $\mathrm{~N} 14-\mathrm{N} 15$ & $1.131(4)$ \\
$\mathrm{C} 3-\mathrm{C} 4$ & $\mathrm{~N} 15-\mathrm{N} 16$ & $1.452(3)$ \\
$\mathrm{C} 3-\mathrm{O} 11$ & $1.514(4)$ & $\mathrm{O} 17-\mathrm{C} 18$ & $1.419(3)$ \\
$\mathrm{C} 3-\mathrm{H} 31$ & $1.452(3)$ & $\mathrm{C} 18-\mathrm{O} 19$ & $1.504(5)$ \\
$\mathrm{C} 4-\mathrm{O} 5$ & 1.005 & $\mathrm{C} 18-\mathrm{C} 21$ & $1.518(4)$ \\
$\mathrm{C} 4-\mathrm{C} 8$ & $1.423(4)$ & $\mathrm{C} 18-\mathrm{C} 22$ & $1.422(3)$ \\
$\mathrm{C} 4-\mathrm{H} 41$ & $\mathrm{O} 19-\mathrm{C} 20$ & 0.976 \\
$\mathrm{O} 5-\mathrm{C} 6$ & $1.538(4)$ & $\mathrm{C} 20-\mathrm{H} 201$ & 0.979 \\
$\mathrm{C} 6-\mathrm{O} 7$ & 0.981 & $\mathrm{C} 20-\mathrm{H} 202$ & 0.984 \\
$\mathrm{C} 6-\mathrm{C} 9$ & $1.433(3)$ & $\mathrm{C} 21-\mathrm{H} 211$ & 0.974 \\
$\mathrm{C} 6-\mathrm{C} 10$ & $1.370(5)$ & $\mathrm{C} 21-\mathrm{H} 212$ & 0.973 \\
$\mathrm{O} 7-\mathrm{C} 8$ & $1.503(6)$ & $\mathrm{C} 21-\mathrm{H} 213$ & 0.980 \\
$\mathrm{C} 8-\mathrm{H} 81$ & $1.486(7)$ & $\mathrm{C} 22-\mathrm{H} 221$ & 0.985 \\
$\mathrm{C} 8-\mathrm{H} 82$ & $1.384(4)$ & $\mathrm{C} 22-\mathrm{H} 222$ &
\end{tabular}




\begin{tabular}{|c|c|c|c|}
\hline C9-H91 & 0.985 & $\mathrm{C} 22-\mathrm{H} 223$ & 0.984 \\
\hline C9-H92 & 0.989 & & \\
\hline $\mathrm{C} 2-\mathrm{C} 1-\mathrm{C} 12$ & $101.8(2)$ & $\mathrm{C} 6-\mathrm{C} 9-\mathrm{H} 93$ & 109.937 \\
\hline $\mathrm{C} 2-\mathrm{C} 1-\mathrm{O} 17$ & $110.6(2)$ & $\mathrm{H} 91-\mathrm{C} 9-\mathrm{H} 93$ & 112.584 \\
\hline $\mathrm{C} 12-\mathrm{C} 1-\mathrm{O} 17$ & $109.0(2)$ & $\mathrm{H} 92-\mathrm{C} 9-\mathrm{H} 93$ & 110.192 \\
\hline $\mathrm{C} 2-\mathrm{C} 1-\mathrm{C} 20$ & $116.9(2)$ & $\mathrm{C} 6-\mathrm{C} 10-\mathrm{H} 101$ & 107.044 \\
\hline $\mathrm{C} 12-\mathrm{C} 1-\mathrm{C} 20$ & $113.8(2)$ & $\mathrm{C} 6-\mathrm{C} 10-\mathrm{H} 102$ & 108.749 \\
\hline $\mathrm{O} 17-\mathrm{C} 1-\mathrm{C} 20$ & $104.8(2)$ & $\mathrm{H} 101-\mathrm{C} 10-\mathrm{H} 102$ & 112.303 \\
\hline $\mathrm{C} 1-\mathrm{C} 2-\mathrm{C} 3$ & $102.9(2)$ & $\mathrm{C} 6-\mathrm{C} 10-\mathrm{H} 103$ & 103.516 \\
\hline $\mathrm{C} 1-\mathrm{C} 2-\mathrm{N} 14$ & $114.3(2)$ & $\mathrm{H} 101-\mathrm{C} 10-\mathrm{H} 103$ & 110.346 \\
\hline $\mathrm{C} 3-\mathrm{C} 2-\mathrm{N} 14$ & $117.3(2)$ & $\mathrm{H} 102-\mathrm{C} 10-\mathrm{H} 103$ & 114.253 \\
\hline $\mathrm{C} 1-\mathrm{C} 2-\mathrm{H} 21$ & 107.763 & $\mathrm{C} 3-\mathrm{O} 11-\mathrm{C} 12$ & $111.1(2)$ \\
\hline $\mathrm{C} 3-\mathrm{C} 2-\mathrm{H} 21$ & 106.585 & $\mathrm{C} 1-\mathrm{C} 12-\mathrm{O} 11$ & $109.7(2)$ \\
\hline $\mathrm{N} 14-\mathrm{C} 2-\mathrm{H} 21$ & 107.444 & $\mathrm{C} 1-\mathrm{C} 12-\mathrm{O} 13$ & $128.4(2)$ \\
\hline $\mathrm{C} 2-\mathrm{C} 3-\mathrm{C} 4$ & $113.8(2)$ & $\mathrm{O} 11-\mathrm{C} 12-\mathrm{O} 13$ & $121.9(3)$ \\
\hline $\mathrm{C} 2-\mathrm{C} 3-\mathrm{O} 11$ & $104.1(2)$ & $\mathrm{C} 2-\mathrm{N} 14-\mathrm{N} 15$ & $115.4(2)$ \\
\hline $\mathrm{C} 4-\mathrm{C} 3-\mathrm{O} 11$ & $108.3(2)$ & $\mathrm{N} 14-\mathrm{N} 15-\mathrm{N} 16$ & $172.0(3)$ \\
\hline $\mathrm{C} 2-\mathrm{C} 3-\mathrm{H} 31$ & 110.457 & $\mathrm{C} 1-\mathrm{O} 17-\mathrm{C} 18$ & $108.8(2)$ \\
\hline $\mathrm{C} 4-\mathrm{C} 3-\mathrm{H} 31$ & 111.708 & $\mathrm{O} 17-\mathrm{C} 18-\mathrm{O} 19$ & $104.9(2)$ \\
\hline $\mathrm{O} 11-\mathrm{C} 3-\mathrm{H} 31$ & 108.048 & $\mathrm{O} 17-\mathrm{C} 18-\mathrm{C} 21$ & $108.6(2)$ \\
\hline $\mathrm{C} 3-\mathrm{C} 4-\mathrm{O} 5$ & $106.4(2)$ & $\mathrm{O} 19-\mathrm{C} 18-\mathrm{C} 21$ & $107.9(2)$ \\
\hline $\mathrm{C} 3-\mathrm{C} 4-\mathrm{C} 8$ & $114.4(2)$ & $\mathrm{O} 17-\mathrm{C} 18-\mathrm{C} 22$ & $109.0(2)$ \\
\hline $\mathrm{O} 5-\mathrm{C} 4-\mathrm{C} 8$ & $104.6(2)$ & $\mathrm{O} 19-\mathrm{C} 18-\mathrm{C} 22$ & $111.4(2)$ \\
\hline $\mathrm{C} 3-\mathrm{C} 4-\mathrm{H} 41$ & 110.832 & $\mathrm{C} 21-\mathrm{C} 18-\mathrm{C} 22$ & $114.6(3)$ \\
\hline $\mathrm{O} 5-\mathrm{C} 4-\mathrm{H} 41$ & 110.245 & $\mathrm{C} 18-\mathrm{O} 19-\mathrm{C} 20$ & $106.8(2)$ \\
\hline $\mathrm{C} 8-\mathrm{C} 4-\mathrm{H} 41$ & 110.109 & $\mathrm{C} 1-\mathrm{C} 20-\mathrm{O} 19$ & $103.3(2)$ \\
\hline $\mathrm{C} 4-\mathrm{O} 5-\mathrm{C} 6$ & $108.4(2)$ & $\mathrm{C} 1-\mathrm{C} 20-\mathrm{H} 201$ & 112.385 \\
\hline $\mathrm{O} 5-\mathrm{C} 6-\mathrm{O} 7$ & $106.2(3)$ & $\mathrm{O} 19-\mathrm{C} 20-\mathrm{H} 201$ & 110.314 \\
\hline $\mathrm{O} 5-\mathrm{C} 6-\mathrm{C} 9$ & $108.9(3)$ & $\mathrm{C} 1-\mathrm{C} 20-\mathrm{H} 202$ & 109.886 \\
\hline $\mathrm{O} 7-\mathrm{C} 6-\mathrm{C} 9$ & $108.9(4)$ & $\mathrm{O} 19-\mathrm{C} 20-\mathrm{H} 202$ & 110.026 \\
\hline $\mathrm{O} 5-\mathrm{C} 6-\mathrm{C} 10$ & $107.6(3)$ & $\mathrm{H} 201-\mathrm{C} 20-\mathrm{H} 202$ & 110.659 \\
\hline $\mathrm{O} 7-\mathrm{C} 6-\mathrm{C} 10$ & $113.0(4)$ & $\mathrm{C} 18-\mathrm{C} 21-\mathrm{H} 211$ & 108.339 \\
\hline $\mathrm{C} 9-\mathrm{C} 6-\mathrm{C} 10$ & $112.0(4)$ & $\mathrm{C} 18-\mathrm{C} 21-\mathrm{H} 212$ & 110.490 \\
\hline $\mathrm{C} 6-\mathrm{O} 7-\mathrm{C} 8$ & $112.1(2)$ & $\mathrm{H} 211-\mathrm{C} 21-\mathrm{H} 212$ & 109.050 \\
\hline $\mathrm{C} 4-\mathrm{C} 8-\mathrm{O} 7$ & $104.4(3)$ & $\mathrm{C} 18-\mathrm{C} 21-\mathrm{H} 213$ & 110.056 \\
\hline $\mathrm{C} 4-\mathrm{C} 8-\mathrm{H} 81$ & 109.437 & $\mathrm{H} 211-\mathrm{C} 21-\mathrm{H} 213$ & 108.699 \\
\hline $\mathrm{O} 7-\mathrm{C} 8-\mathrm{H} 81$ & 109.119 & $\mathrm{H} 212-\mathrm{C} 21-\mathrm{H} 213$ & 110.158 \\
\hline $\mathrm{C} 4-\mathrm{C} 8-\mathrm{H} 82$ & 113.777 & $\mathrm{C} 18-\mathrm{C} 22-\mathrm{H} 221$ & 114.558 \\
\hline $\mathrm{O} 7-\mathrm{C} 8-\mathrm{H} 82$ & 109.278 & $\mathrm{C} 18-\mathrm{C} 22-\mathrm{H} 222$ & 113.225 \\
\hline $\mathrm{H} 81-\mathrm{C} 8-\mathrm{H} 82$ & 110.606 & $\mathrm{H} 221-\mathrm{C} 22-\mathrm{H} 222$ & 107.285 \\
\hline C6-C9-H91 & 105.028 & $\mathrm{C} 18-\mathrm{C} 22-\mathrm{H} 223$ & 107.279 \\
\hline C6-C9-H92 & 109.043 & $\mathrm{H} 221-\mathrm{C} 22-\mathrm{H} 223$ & 106.580 \\
\hline $\mathrm{H} 91-\mathrm{C} 9-\mathrm{H} 92$ & 109.895 & $\mathrm{H} 222-\mathrm{C} 22-\mathrm{H} 223$ & 107.508 \\
\hline
\end{tabular}

